\title{
Is there a Volume-Outcomes Relationship for Patients Undergoing Surgical Management of Acute Stanford Type A Aortic Dissection?
}

\section{Mimi Borrelli ${ }^{1}$, Christopher Young ${ }^{2}$ and Rizwan Attia ${ }^{1^{*}}$}

${ }^{1}$ Department of Cardiothoracic Surgery, King's College Hospital, London, UK

${ }^{2}$ Department of Cardiothoracic Surgery, Guy's and St Thomas' Hospital, London, UK

\begin{abstract}
Aim: To study the world literature on patients undergoing surgical repair of acute Stanford type A aortic dissection (ATAAD). We assessed if the volume or experience of the center or surgeon affects outcomes.

Methods: Literature was reviewed using the OVID platform for Medline $₫$ and Pubmed from 1966-2017. 51 papers were identified of which 12 studies met the inclusion criteria and represented the highest level of evidence to answer the research question.

Results: Retrospective cohort analysis of 1550 UK patients operated on by 249 surgeons using national audit data found low volume surgeons (LVS) had higher in-hospital mortality vs high volume surgeons (HVS) (19.3\% vs $12.6 \%, P=0.015$, OR: $0.853 \mathrm{Cl}: 0.733-0.992, \mathrm{P}=0.039$ ), but no relationship between mortality and center volume. Five large retrospective studies using the United States (US) Nationwide Inpatient Sample (NIS) database looked at 29292 cases. Higher-volume centers (HVC) were associated with lower mortality following ATAAD repair vs lower-volume centers (LVC) $(27.5 \%$ vs $16.4 \%, \mathrm{P}<0.001 ; 34 \%$ vs $25 \%, \mathrm{P}=0.003 ; 23.4 \%$ vs $12.1 \%, \mathrm{P}=0.014$; $12.6 \%$ vs $23.9 \%, P=0.013)$. A single-center cohort study in Germany, UK and three in the US reported decreased mortality after introduction of a specialized team/protocol for ATAAD repair $(34.5 \%$ vs $8 \%, \mathrm{P}<0.001 ; 33.9 \%$ vs $2.8 \%, \mathrm{P}<0.0001 ; 33.9 \%$ vs $7.7 \%, \mathrm{P}<0.0001 ; 22.9 \%$ vs $9.7 \%, \mathrm{P}=0.002 ; 30.8 \%$ vs $9.7 \%, \mathrm{P}=0.014$ ).
\end{abstract}

Conclusion: There is moderate evidence of a volume-outcome relationship for ATAAD surgery. This may be driven by surgeon or institutional volume. There is stronger evidence that establishing specialized multidisciplinary teams and standardized protocols reduces morbidity and mortality.

Keywords: Aortic dissection; Surgery; Volume; Outcomes; Morbidity; Mortality

\section{Introduction}

ATAAD is a surgical emergency associated with high mortality and morbidity. $50 \%$ of patients die in the first 24 hours [1-3], and $90 \%$ of patients left untreated die within a week [4]. The surgical repair of ATAAD is a challenging, high-risk procedure, and involves replacing the intimal dissection of the ascending aorta and/or arch with a prosthetic graft. Reported surgical mortality ranges from $15 \%$ to $32 \%$ [5-7]. Furthermore, ATAAD is rare, with an estimated 5 to 30 million cases per year [8], and mortality outcomes following ATAAD repair may be further impacted by the volumes seen by the center and the operating surgeons. A volume-outcomes relationship has been shown in other forms of cardiothoracic surgery including coronary artery bypass grafting [9], pediatric cardiac surgery [10] and in surgical procedures such as coronary angioplasty [11]. Transfer to a highvolume center specializing in aortic surgery can lengthen journey times in unwell patients, risking their deterioration during transfer and delaying time-critical surgery. This risk may be counteracted by the potential benefit of experience and facilities available at high-volume centers including multidisciplinary teams of cardiothoracic surgeons, consultant anesthetists, specialist nurses, the protocols and intensive care equipped to provide optimal post-operative cardiovascular support [12]. In major trauma, an equally time critical situation, transporting all trauma patients regardless of clinical stability to a highly specialist "major trauma" center is associated with significantly decreased mortality [13]. Creating specialist centers concentrates the cases of ATAAD and enables research, treatment advances and exposure and training in a risky and complex procedure. This paper studies the mortality and morbidity of patients with ATAAD treated with surgical repair in high-volume centers (HVC) compared to low volume centers (LVC).

\section{Methods}

OVID MEDLINE ${ }^{\circledR}$ and PubMed were searched from 1966 to December 2017, using the terms: (ortic dissection) AND (volume) AND (mortality OR morbidity OR outcomes). Two of the researchers (MD and RA) independently screened each of the 51 articles generated for inclusion against the inclusion and exclusion criteria (Table 1). Articles were included if they reported the mortality outcomes of patients who underwent operative repair for ATAAD, and if there was a mortality comparison between either centers and/or surgeons of different volumes. The final list of generated articles by each researcher was cross-checked. The same two researchers then independently extracted data from articles which met the inclusion criteria into a preformatted excel database. Data extracted included: The authors, date, journal, country of publication, study type, volume categorization of surgeon and/or hospital and relevant outcomes. All data extracted was cross-checked. The articles representing the highest level of evidence were included in this study.

*Corresponding author: Rizwan Attia, Department of Cardiothoracic Surgery, King's College Hospital, London, SE5 9RS, UK, Tel: 4402032993871; Fax: 4402032993433; E-mail: rizwanattia@doctors.org.uk

Received February 22, 2018; Accepted March 14, 2018; Published March 16 2018

Citation: Borrelli M, Young C, Attia R (2018) Is there a Volume-Outcomes Relationship for Patients Undergoing Surgical Management of Acute Stanford Type A Aortic Dissection? J Cardiovasc Dis Diagn 6: 311. doi: 10.4172/23299517.1000311

Copyright: ( 2018 Borrelli M, et al.. This is an open-access article distributed under the terms of the Creative Commons Attribution License, which permits unrestricted use, distribution, and reproduction in any medium, provided the original author and source are credited. 


\section{Results and Discussion}

12 articles met the inclusion criteria (Figure 1), and relevant article details are presented in Supplementary Table 1. A large retrospective cohort analysis conducted in the United Kingdom (UK) by Bashir et al. [14] used the National Institute for Cardiovascular Outcomes Research database to identify 1500 patients who had ATAAD repaired across 41 hospitals by 249 different cardiac surgeons. Surgeons were categorized as low-volume (LVS, with a mean annual volume (MAV) of $<4$ ) or high-volume (HVS, MAV $\geq 4$ ), a cut-off value selected as clinically meaningful after introductory analysis. The overall in-hospital mortality was $18.3 \%$. LVS had higher in-hospital mortality compared to HVS (19.3\% vs $12.6 \%, \mathrm{P}=0.015$, OR: 0.853 CI: 0.733-0.992, $\mathrm{P}=0.039$ ), however mortality differences rebased beyond 90 days. There was little relationship between mortality and hospital volume and a wide variation of in-hospital mortality around the country.

Five large retrospective analyzes were conducted using the United States (US) Nationwide Inpatient Sample (NIS) database. In the most recent of these, Chikwe et al. [15] analyzed institution and surgeon volumes as predictors of operative mortality in patients $(\mathrm{N}=5184)$ with acute aortic dissection (AD). Data were collected from the United States (US) National Inpatient Sample (NIS) database (2003-2008), which precluded separation of $\mathrm{AD}$ types $\mathrm{A}$ and $\mathrm{B}$. Annual $\mathrm{AD}$ repair volumes were calculated (total repairs/years surveyed) and categorized into quartiles for surgeons and institutions to ensure equal numbers in each quartile. LVC ( $\leq 3$ repairs/year) had significantly increased mortality vs HVC (>13/year); $27.4 \%$ vs $16.4 \%, \mathrm{P}<0.001$; OR:2.21, CI:1.72-2.86, $\mathrm{P}<0.001$. LVS $(<1 /$ year) had higher operative mortality vs HVS $(\geq 5 /$ year); $27.5 \%$ vs $17.0 \%, \mathrm{P}<0.001$; OR:1.78, CI:1.39-2.29, $\mathrm{P}<0.001$.

Knipp et al. [16] also used data from the NIS database (1995-2003) to analyze center volumes as a predictor of mortality in 3013 patients with acute $\mathrm{AD}$ (types $\mathrm{A}$ and $\mathrm{B}$ ). There was insufficient data in this time period to calculate surgeon-specific volume. Hospitals were stratified into LVC, intermediate volume center (IVC) and HVC based on annual caseload across the study period $(<1.0,1.0-2.5,>2.5)$. Mortality was $34 \%$ in LVC, and despite identical mortality rates in IVC and HVC (25\%), 'volume' entered as a single factor significantly predicted mortality in univariate analysis $(\mathrm{P}<0.001)$ but was not an independent predictor of mortality in multivariate analysis. Annual hospital procedural volume was inversely correlated with mortality $(\mathrm{R}=-0.158, \mathrm{P}<0.001)$.

In a similar study Iribarne et al. [17] used the NIS database (20052008 ) to analyze institutional volume as a predictor of mortality in patients $(\mathrm{N}=1230)$ undergoing acute $\mathrm{AD}$ repair (types $\mathrm{A}$ and $\mathrm{B})$. Annual institutional $\mathrm{AD}$ repair volume demonstrated three clusters across the study period; $H V C \geq 11$, IVC $6-10, \mathrm{LVC} \leq 5$. Multivariate regression revealed a significant effect of hospital volume on mortality rates (LVC $23.4 \%$ vs IVC $20.1 \%$ vs HVC $12.1 \%, \mathrm{P}=0.014$ ), number of patients with no complications (HVC $63.7 \%$ vs $52.3 \%$ of LVC patients, $\mathrm{P}=0.044$ ), and discharge disposition (greatest home discharges in $\mathrm{HVC}, \mathrm{P}=0.011$ ).

\begin{tabular}{|c|}
\hline Articles were included if they reported \\
\hline Mortality outcomes \\
\hline Patients underwent operative repair for Type A aortic dissection \\
\hline A mortality comparison between centres and/or surgeons of different volumes \\
\hline Articles were excluded if they were \\
\hline Looking at any other aortic pathology (aneurysms/ type B dissections) \\
\hline If the articles were not presenting original research \\
\hline Articles presenting lower level of evidence (level 3-5) \\
\hline Table 1: Exclusion/inclusion criteria.
\end{tabular}

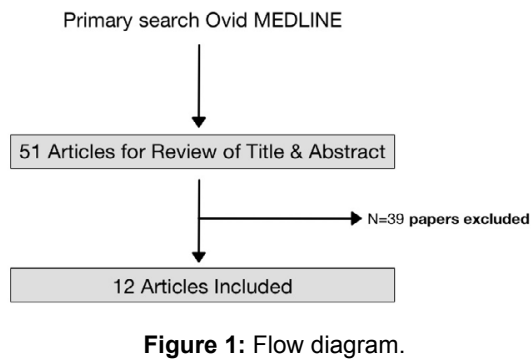

Merlo et al. [18] analyzed center volume and admission type as predictors of mortality in patients $(\mathrm{N}=1507)$ undergoing emergent open acute $\mathrm{AD}$ repair (Type $\mathrm{A}$ and $\mathrm{B}$ ), again using the NIS database (2004-2008). Following previous work, [17] centers were categorized into HVC, IVC and LVC according to annual caseload $(<5,6-10, \geq 11$ respectively). Data were categorized as transfer (TA) and direct (DA) admissions, to control for survival bias improving mortality outcomes at HVC. Univariate analysis indicated mortality was lower at HVC vs IVC ( $12.6 \%$ vs $20.6 \%, \mathrm{P}=0.001)$, lower at HVC vs LVC ( $12.6 \%$ vs $23.9 \%$, $\mathrm{P}=0.025)$, and lower for $\mathrm{DA}$ at $\mathrm{HVC}$ vs $\mathrm{LVC}(10.6 \%$ vs $24.0 \%, \mathrm{P}=0.013)$, but did not differ for TA. In multivariate analysis admission to LVC was an independent risk of mortality vs HVC (OR:2.06 CI: 1.25-3.38, $\mathrm{P}=0.004)$.

In the final of the 5 studies using the NIS database Zimmerman et al. [19] analyzed mortality outcomes following AD repair according to center volume (2003-2012). Center volume was categorized into HVC and non-HVC; HVC were those in the highest decile for AD surgical repairs $(\geq 6)$ during the year of admission in the NIS. AD patients either had medical management $(\mathrm{N}=7964)$, thoracic endovascular aortic repair $(\mathrm{N}=1417)$, open surgical repair of type A (TASR, $\mathrm{N}=3253$ ) or type $\mathrm{B}$ AD $(\mathrm{N}=3007)$. In multivariate analysis admission to a $\mathrm{HVC}$ was associated with a decreased mortality rate for type B AD (OR:0.55; CI:0.4-0.7, $\mathrm{P}<0.01$ ), but not TASR.

One single-center study analyzed surgeon volume as a predictor of mortality. Murzi et al. [20] compared mortality outcomes of 35 ATAAD repairs performed by two HVS, to 79 performed by six LVS in a single center in Italy. The range of surgical experience across all 8 surgeons was 28-238 repairs, but the method for categorizing surgeons and the individual surgeon caseloads were not reported. There was no difference in mortality or morbidity rates between HVS and LVS.

Single-center cohort studies have been conducted in Germany, the UK and three have been conducted in the US. All have reported decreased mortality after introduction of a specialized team/protocol for aortic dissection repair. Beller et al. [21] compared outcomes of ATAAD repairs at a single US center before $(\mathrm{N}=39,2005-2009)$ and after $(\mathrm{N}=62,2010-2014)$ the introduction of a multi-disciplinary Aortic Surgery Team (AST) and standardized management protocols. PreAST $>5 \%$ repairs were performed by 5 surgeons. Post-AST 2 surgeons performed $>5 \%$ repairs with the highest-volume surgeon performing $74.2 \%$. Post-AST there was a reduction in mortality (30.8\% vs $9.7 \%$, $\mathrm{P}=0.014)$, acute renal failure, $(38.5 \%$ vs $17.7 \%, \mathrm{P}=0.034)$, prolonged ventilation (69.2 vs $32.2 \%, \mathrm{P}<0.001)$, and tracheostomy $(25.6 \%$ vs $3.2 \%$, $\mathrm{P}=0.001)$.

Lenos et al. [22] analyzed mortality outcomes of ATAAD repairs performed by surgeons belonging (AT; $\mathrm{N}=75$ ) or not belonging to the Aortic Team (non-AT, N=87) in a single center in Germany (20022013). Compared to non-AT patients, AT patients had fewer adverse outcomes ( $8 \%$ vs $34.5 \%, \mathrm{P}<0.0001)$, lower mortality rates ( $4 \%$ vs $21.8 \%$, 
$\mathrm{P}<0.001)$, and lower rates of permanent neurological deficit $(2.7 \% \mathrm{vs}$ $11.5 \%, \mathrm{P}=0.03)$. Multivariate analysis identified surgery not-performed by the AT as the strongest independent predictor of adverse outcomes (OR:14.1, CI:3.5-55.6, P<0.001).

Andersen et al. [23] compared outcomes of ATAAD repairs in a single US center (1999-2011) before $(\mathrm{N}=56)$ and after $(\mathrm{N}=72)$ the establishment of a Thoracic Aortic Surgery Programme (TASP). PreTASP 11 surgeons performed all repairs (average annual surgeon volume $2 \pm 1.3$, annual institutional volume 9). Post-TASP two principle TASP surgeons performed $97 \%$ of repairs (annual surgeon volumes 9.7 and 4.0, annual institutional volume 11). Post-TASP operative mortality rates dropped $(33.9 \%$ vs $2.8 \%, \mathrm{P}<0.0001)$, and the 5 -year survival was higher ( $55 \%$ vs $85 \%, \mathrm{P}=0.0017$ ), postoperative complications lower $(19.6 \%$ vs $4.2 \%, \mathrm{P}=0.005)$ and hospital stay was reduced $(12 \pm$ 12 vs $10 \pm 12$ days, $\mathrm{P}=0.05)$ were improved. In 2013 two new TASP surgeons and a protocol for triage, diagnostic and transfer (RACE-AD) were introduced [24]. The average annual institutional repair volume increased from 2005-2012 to 2013-2015 ( $12 \pm 2$ vs $22 \pm 6, \mathrm{P}=0.004$ ), but average surgeon volumes, and mortality benefits remained improved 10 -years post $(\mathrm{N}=156)$ vs pre-TASP $(\mathrm{N}=56)(7.7 \%$ vs $33.9 \%, \mathrm{P}<0.0001)$.

Sales et al. [25] analyzed mortality following thoracic aortic surgery before $(\mathrm{N}=157)$ and after $(\mathrm{N}=175)$ formation of a Center for Aortic Surgery (CTA) in a single center in Brazil. Pre- vs Post CTA there was a decrease in overall mortality $(22.9 \%$ vs $9.7 \%, \mathrm{P}=0.008)$, mortality following type $\mathrm{B} A D$ repair ( $37.5 \%$ vs $18.7 \%, \mathrm{P}=0.038$ ), all post-operative complications $(56.7 \%$ vs $38.9 \%, \mathrm{P}=0.001$ ), length of hospital length stay (6.4 \pm 7.2 vs $4.8 \pm 8.4$ days, $\mathrm{P}=0.001$ ), but not mortality following ATAAD repair.

\section{What is the Take Home Message for the Clinician?}

There is evidence for a volume-outcomes relationship in surgery for ATAAD. Mortality and morbidity benefits were found in higher-volume centers and in higher-volume surgeons. Specialized aortic surgery programs employing perioperative protocols within multidisciplinary teams with appropriate surgical supervision can lead to increased volumes and result in consistent benefits in outcomes for acute aortic dissection.

\section{Conclusion}

There is moderate evidence from retrospective observational studies for a volume-outcomes relationship in surgery for AD. This which may be driven by surgeon and institutional volume. There is stronger evidence that specialized aortic surgery programs, employing perioperative protocols, HVS and multidisciplinary aortovascular teams, lead to improved outcomes for acute AD.

\section{Conflict of Interest}

None declared.

\section{Funding}

No funding was received.

\section{Disclosures}

None

\section{References}

1. Gooiker GA, van Gijn W, Wouters MW, Post PN, Van De Velde C, et al. (2011) Systematic review and meta-analysis of the volume-outcome relationship in pancreatic surgery. Brit J Surg 98: 485-494.
2. Trinh QD, Bjartell A, Freedland SJ, Hollenbeck BK, Hu JC, et al. (2013) A systematic review of the volume-outcome relationship for radical prostatectomy. Euro Urol 64: 786-798.

3. Kar-mun CW, Schneider JI (2009) High-risk chief complaints I: chest pain-the big three. Emergency medicine clinics of North America 27: 685-712.

4. Hagan PG, Nienaber CA, Isselbacher EM, Bruckman D, Karavite DJ, et al. (2000) The International Registry of Acute Aortic Dissection (IRAD): new insights into an old disease. Jama 283: 897-903.

5. Trimarchi S, Nienaber CA, Rampoldi V, Myrmel T, Suzuki T, et al. (2005) Contemporary results of surgery in acute type A aortic dissection: The International Registry of Acute Aortic Dissection experience. J Thoracic Cardiovasc Surg 129: 112-122.

6. Booher AM, Isselbacher EM, Nienaber CA, Trimarchi S, Evangelista A, et al. (2013) The IRAD classification system for characterizing survival after aortic dissection. Am J Med 126: 730. e19-730. e24

7. Rampoldi V, Trimarchi S, Eagle KA, Nienaber CA, Oh JK, et al. (2007) Simple risk models to predict surgical mortality in acute type A aortic dissection: the International Registry of Acute Aortic Dissection score. Ann Thoracic Surg 83: $55-61$

8. Khan IA, Nair CK (2002) Clinical, diagnostic, and management perspectives of aortic dissection. Chest J 122: 311-328.

9. Hannan EL, Wu C, Ryan TJ, Bennett E, Culliford AT, et al. (2003) Do hospitals and surgeons with higher coronary artery bypass graft surgery volumes still have lower risk-adjusted mortality rates? Circulation 108: 795-801.

10. Hannan EL, Racz M, Kavey RE, Quaegebeur JM, Williams R (1998) Pediatric cardiac surgery: the effect of hospital and surgeon volume on in-hospital mortality. Pediatrics 101: 963-969.

11. Hannan EL, Racz M, Ryan TJ, McCallister BD, Johnson LW, et al. (1997) Coronary angioplasty volume-outcome relationships for hospitals and cardiologists. Jama 277: 892-898.

12. Haq J, Gately F, Bentley R (2017) Implementation of an oral and maxillofacia surgery trauma team in a major trauma centre. Brit $J$ Oral Maxillofacial Surg 55: 396-399.

13. Davenport R, Tai N, West A, Bouamra O, Aylwin C, et al. (2010) A major trauma centre is a specialty hospital not a hospital of specialties. Brit J Surg 97: 109117

14. Bashir M, Harky A, Fok M, Shaw M, Hickey GL, et al. (2017) Acute type A aortic dissection in the United Kingdom: Surgeon volume-outcome relation. J Thoracic Cardiovasc Surg 154: 398-406.e1.

15. Chikwe J, Cavallaro P, Itagaki S, Seigerman M, DiLuozzo G, et al. (2013) National outcomes in acute aortic dissection: influence of surgeon and institutional volume on operative mortality. Ann Thoracic Surg 95: 1563-1569.

16. Knipp BS, Deeb GM, Prager RL, Williams CY, Upchurch GR, et al. (2007) A contemporary analysis of outcomes for operative repair of type $A$ aortic dissection in the United States. Surgery 142: 524-528. e1.

17. Iribarne A, Milner R, Merlo AE, Singh A, Saunders CR, et al. (2015) Outcomes following emergent open repair for thoracic aortic dissection are improved at higher volume centers. J Cardiac Surg 30: 74-79.

18. Merlo AE, Chauhan D, Pettit C, Hong KN, Saunders CR, et al. (2016) Outcomes following emergent open repair for thoracic aortic dissection are improved at higher volume centers in direct admissions and transfers. J Cardiothoracic Surg 11: 118.

19. Zimmerman KP, Oderich G, Pochettino A, Hanson KT, Habermann EB, et al. (2016) Improving mortality trends for hospitalization of aortic dissection in the National Inpatient Sample. J Vasc Surg 64: 606-615.e1.

20. Murzi M, Miceli A, Di Stefano G, Cerillo AG, Kallushi E, et al. (2015) Enhancing quality control and performance monitoring in thoracic aortic surgery: a 10-year single institutional experience. Euro J Cardio-Thoracic Surg 47: 608-615.

21. Beller JP, Scheinerman JA, Balsam LB, Ursomanno P, DeAnda Jr A (2015) Operative strategies and outcomes in type a aortic dissection after the enactment of a multidisciplinary aortic surgery team. innovations: technology and techniques in Cardiothoracic and Vascular Surgery 10: 410-415.

22. Lenos A, Bougioukakis P, Irimie V, Zacher M, Diegeler A, et al. (2014) Impact of surgical experience on outcome in surgery of acute type $A$ aortic dissection. Euro J Cardio-Thoracic Surg 48: 491-496. 
Citation: Borrelli M, Young C, Attia R (2018) Is there a Volume-Outcomes Relationship for Patients Undergoing Surgical Management of Acute Stanford Type A Aortic Dissection? J Cardiovasc Dis Diagn 6: 311. doi: 10.4172/2329-9517.1000311

Page 4 of 4

23. Andersen ND, Ganapathi AM, Hanna JM, Williams JB, Gaca JG, et al. (2014) Outcomes of acute type a dissection repair before and after implementation of a multidisciplinary thoracic aortic surgery program. J Am College Cardiol 63 1796-1803.

24. Andersen ND, Benrashid E, Ross AK, Pickett LC, Smith PK, et al. (2016) The utility of the aortic dissection team: outcomes and insights after a decade of experience. Ann Cardiothoracic Surg 5: 194-201.

25. Sales MdC, Frota Filho JD, Aguzzoli C, Souza LD, Rösler ÁM, et al. (2014) Aortic Center: specialized care improves outcomes and decreases mortality. Revista Brasileira de Cirurgia Cardiovascular 29: 494-504. 